\title{
Why fundamentals of endoscopic surgery (FES)?
}

\author{
Jeffrey W. Hazey $\cdot$ Jeffrey M. Marks $\cdot$ John D. Mellinger $\cdot$ Thadeus L. Trus $\cdot$ \\ Bipan Chand · Conor P. Delaney • Brian J. Dunkin • Robert D. Fanelli • \\ Gerald M. Fried · Jose M. Martinez · Jonathan P. Pearl • Benjamin K. Poulose • \\ Lelan F. Sillin · Melina C. Vassiliou $\cdot$ W. Scott Melvin
}

Received: 25 April 2013/Accepted: 30 August 2013/Published online: 7 December 2013

(C) Society of American Gastrointestinal and Endoscopic Surgeons 2013

As flexible endoscopy has moved into the mainstream, gastroenterologists have embraced many of the skills and techniques particular to this modality of diagnosis and intervention. Their adoption of flexible endoscopic technology and training, and the lack of enthusiasm for endoscopic therapy potentials by surgeons, has left many surgical residents and practicing surgeons deficient in endoscopic skills. As a result, education of surgical residents in flexible endoscopy has lagged and training of surgical residents in flexible endoscopy is increasingly coming under scrutiny and has become an area of debate. The medical literature and practice guidelines are replete with articles from surgeons and gastroenterologists debating the appropriate education and training in flexible endoscopy. Both surgical and gastroenterology professional societies have published guidelines for training in flexible endoscopy. These guidelines are often at odds with each other, citing opposing literature supporting their position on appropriate criteria for training in basic upper and lower endoscopy [1-4]. Flexible endoscopy is a critical element of any general surgeon's and colorectal surgeon's practice. In 2007, $74 \%$ of rural surgeons performed more than 50 flexible endoscopic procedures each year, with $42 \%$ of rural surgeons performing more than 200 flexible

Taskforce Members are listed in Appendix.

J. W. Hazey $(\bowtie) \cdot$ J. M. Marks · J. D. Mellinger .

T. L. Trus · B. Chand - C. P. Delaney - B. J. Dunkin ·

R. D. Fanelli · G. M. Fried - J. M. Martinez .

J. P. Pearl · B. K. Poulose · L. F. Sillin .

M. C. Vassiliou - W. S. Melvin

Section of Minimally Invasive Surgery, Department of Surgery,

The Wexner Medical Center, The Ohio State University, N708

Doan Hall, 410 West Tenth Avenue, Columbus, OH 43210, USA

e-mail: jeffrey.hazey@osumc.edu endoscopic procedures annually [5]. In a 2010 report on rural, under-served areas that lack gastroenterology services, $39.8 \%$ of an American general surgeons' practice comprises flexible endoscopic procedures [6]. In Canada, surgeons were found to be the primary providers of flexible endoscopic services in smaller urban and rural areas [7].

The American Board of Surgery (ABS) has begun to address the training inequity that exists between general surgery residents and gastroenterology fellows [8]. In an effort to ensure surgical residents are fully trained and competent in flexible endoscopy, the ABS has not only increased the minimum requirements for training general surgery residents in flexible endoscopy but has also undertaken the task of formalizing a flexible endoscopy curriculum for its residents. Currently, the ABS and Residency Review Committee (RRC) recommend 35 upper endoscopic procedures and 50 colonoscopies as the minimum number of procedures to be performed by surgical residents. The Society of American Gastrointestinal and Endoscopic Surgeons (SAGES) and the ABS have long espoused that numbers do not ensure competency in surgical or endoscopic procedures. This position is fully supported by data. In 2004, the SAGES esophagogastroduodenoscopy (EGD) Outcomes Study Group prospectively reviewed 3,525 EGDs performed by surgeons, showing a high degree of success with low morbidity. There was no correlation between experience (i.e. number of cases performed) and completion rates or major complications [9]. A similar trial by the SAGES Colonoscopy Study Outcomes Group prospectively reviewed 13,580 colonoscopies performed by surgeons and found no correlation between experience and complications, with an acceptable success rate. The investigators noted that a minimum of 50 colonoscopies with 100 performed annually showed a significant improvement in completion rates 
[10]. In a separate trial of 14,064 patients who underwent colonoscopy within 3 years of a diagnosis of a colorectal cancer, surgeons had equivalent performance with respect to missed lesions. Endoscopy volume was not associated with missed lesions [11]. In contrast with the notion that general surgeons are inadequately trained, a study of specific metrics of performance in 5,237 colonoscopies by general surgeons, colorectal surgeons, and gastroenterologists also found no differences among these specialties [12]. Despite this strong supporting literature, a validated educational tool that addresses training and basic endoscopic knowledge is needed, especially for surgical trainees and practicing general surgeons.

The two leading societies with an interest in flexible endoscopy, SAGES and the American Society for Gastrointestinal Endoscopy (ASGE), are committed to training residents and fellows in flexible endoscopy. Yet, no validated training tool in flexible endoscopy exists today. In an effort to facilitate fundamental and basic training in flexible endoscopy, SAGES and the ABS are producing a comprehensive curriculum to ensure surgical residents and surgeons are educated and have basic skills in flexible endoscopy. This is in part a response to the notion that residents are inadequately trained in flexible endoscopy. SAGES developed the Fundamentals of Endoscopic Surgery $^{\mathrm{TM}}$ (FES) as one part of the proposed ABS flexible endoscopy curriculum. FES is an educational tool very similar to the Fundamentals of Laparoscopic Surgery (FLS). It includes a comprehensive web-based didactic component of flexible endoscopy, a written multiple choice test of knowledge (cognitive component), and a fivemodule virtual reality skills exam (hands-on component), all designed to teach and validate basic endoscopic knowledge and skills. The didactic component is webbased and includes 12 modules of educational content. FES has been developed with validity evidence for a high-stakes test to identify a minimum-level-of-proficiency candidate. FES is the first educational tool with validity evidence combining knowledge and skill for flexible endoscopy.

So why should we develop and care about FES? Ultimately, the goal of SAGE is to improve the quality of care delivered to all of our patients in the safest possible manner. Ensuring surgeons are adequately trained in basic and fundamental flexible endoscopic skills means patients can be assured their surgeon has had didactic and hands-on training in basic flexible endoscopy. The institution of a program of didactic and hands-on skills impacts patient safety. It is reasonable to assume FES will follow the course of FLS, and the skills developed on the endoscopic simulator used in FES education will translate to improved performance. Data published in January 2010 show that skills developed on the FLS simulator improves laparoscopic performance in the operating room [13]. FLS, and presumably FES, can be taught at all levels: medical school students, residents, and attending surgeons. In fact, early training in laparoscopic skills has translated into improved performance in early-level surgical residents [14]. Skill benefits may be realized early in surgical education, which will improve efficiency of training, quality, and patient safety. Once FES training has been implemented, the surgical community must study and measure its intended effects on quality of patient care and safety. The success of FLS has paved the path for the successful implementation of FES. With the ABS adoption of FLS training as a requirement for all surgeons before board eligibility, it is our hope the ABS will take a similar path with FES. Adoption and implementation of FLS after the ABS mandate has been an unmitigated success [15, 16]. In a 2010 article, the Controlled Risk Insurance Company of Harvard's Risk Management Foundation (CRICO/RMF) sponsored an FLS course for its surgeons with the anticipated result of advancing competency and patient safety [17]. As the track record accumulates, we anticipate similar benefits with FES, as both training programs have undergone evaluation for validity evidence using rigorous psychometric techniques.

Flexible endoscopy is a central component of surgical history and is necessary for quality, comprehensive care of the surgical patient. Surgeons are uniquely positioned to perform upper endoscopy as part of the work-up of patients prior to surgical intervention. Additionally, intra-operative upper endoscopy is essential for surgeons performing foregut procedures to identify adequacy of therapy delivered in the operating room. Similarly, when performing flexible endoscopy post-operatively, surgeons are uniquely positioned to understand the anatomic changes necessary to deliver quality care. The ability to endoscopically localize and plan operative therapy by colorectal surgeons is essential to the practice of colorectal surgery. It is our duty to maintain our involvement in flexible endoscopic techniques, especially in light of the fact that new procedures (e.g. peroral endoscopic myotomy [POEM]) may replace traditional open and laparoscopic techniques.

With the changing healthcare environment, we can no longer justify training and education with the ideology of 'that is just how it works' or 'it is what we have done in the past'. Today, we need to develop and study our flexible endoscopy curriculum to ensure the highest quality patient care and safety.

Acknowledgments Prepared in conjunction with the Fundamentals of Endoscopic Surgery (FES) Committee within the Society of American Gastrointestinal and Endoscopic Surgeons (SAGES).

Disclosures Jeffrey W. Hazey, Jeffrey M. Marks, John D. Mellinger, Thadeus L Trus, Bipan Chand, Conor P. Delaney, Brian J. Dunkin, Robert D. Fanelli, Gerald M. Fried, Jose M. Martinez, 
Jonathan P. Pearl, Benjamin K. Poulose, Lelan F. Sillin, Melina C. Vassiliou, and W. Scott Melvin have no conflicts of interest or financial ties to disclose.

\section{Appendix}

Fundamentals of Endoscopic Surgery (FES) Committee Members

Gina L. Adrales, Mehran Anvari, Maurice E. Arregui, Guilherme R. Campos, Patrice R. Carter, Bipan Chand, Edward Gerald Chekan, Chike V. Chukwumah, Ananya Das, Conor P. Delaney, Brian J. Dunkin, C. Neal Ellis, James C. Ellsmere, Robert D. Fanelli, Ashley L. Faulx, Erika K. Fellinger, Kenneth A. Forde, Gerald M. Fried, Karen Galvan, Denise W. Gee, Keith Steven Gersin, Jeffrey W. Hazey, Blair A. Jobe, Rebekah S. Kim, James R. Korndorffer, Jeffrey M. Marks, John H. Marks, Michael R. Marohn, Jose M. Martinez, Brent D. Matthews, David A. McClusky, Michael F. McGee, Ozanan Ricardo de Oliveira Meireles, John D. Mellinger, Renee Minjarez, Kalyana Nandipati, Craig Olson, Raymond P. Onders, Adrian E. Park, Eric M. Pauli, Jonathan P. Pearl, Melissa Susan Phillips, Benjamin K. Poulose, Nitin (Nick) J. Rangnekar, William O. Richards, E. Matthew Ritter, Byron Fernando Santos, David E. Scheeres, Daniel J. Scott, Ross D. Segan, Lelan F. Sillin, C. Daniel Smith, Nathaniel J. Soper, Lee L. Swanstrom, Klaus Thaler, Thadeus L. Trus, David R. Urbach, Kent R. VanSickle, Melina C. Vassiliou, Vic Velanovich, Gary C. Vitale, Eric T. Volckmann, Jonathan P. Walker, R. Larry Whelan, Patrick Yachimski, Debbie F. Youngelman.

\section{References}

1. SAGES Guidelines Committee (2008) Granting of privilege for gastrointestinal endoscopy. Surg Endosc 22:1349-1352

2. Eisen GM, Baron TH, Dominitz JA et al (2002) Methods of granting hospital privileges to perform gastrointestinal endoscopy. Gastrointest Endosc 55:780-783
3. Wexner SD, Litwin D, Cohen J et al (2002) Principles of privileging and credentialing for endoscopy and colonoscopy. Gastrointest Endosc 55:145-148

4. ASGE, AASLD, ACG and AGA statement regarding the ABS mandate for surgery resident training in endoscopy (2011). http:// www.asge.org/uploadedFiles/Pressroom/JointSocietyABSTraining Statement2011.pdf. Accessed 23 Apr 2013

5. Zuckerman R, Doty B, Bark K et al (2007) Rural versus non-rural differences in surgeon performed endoscopy: results of a national survey. Am Surg 73(9):903-905

6. Harris JD, Hosford CC, Sticca RP (2010) A comprehensive analysis of surgical procedures in rural surgery practices. Am J Surg 200(6):820-825

7. Hilsden RJ, Tepper J, Moayyedi P et al (2007) Who provides gastrointestinal endoscopy in Canada? Can $\mathrm{J}$ Gastroenterol 21(12):843-846

8. The American Board of Surgery (2011) ABS statement on GI endoscopy. http://www.absurgery.org/default.jsp?newsgiresponse. Accessed 23 Apr 2013

9. Reed WP, Kilkenny JW, Dias CE et al (2004) A prospective analysis of 3525 esophagogastroduodenoscopies performed by surgeons. Surg Endosc 18:11-21

10. Wexner SD, Garbus JE, Singh JJ, SAGES Colonoscopy Study Outcomes Group (2001) A prospective analysis of 13,580 colonoscopies. Reevaluation of credentialing guidelines. Surg Endosc $15: 251-261$

11. Baxter NN, Sutradhar R, Forbes SS et al (2011) Analysis of administrative data finds endoscopist quality measures associated with postcolonoscopy colorectal cancer. Gastroenterology 140:65-72

12. Mehran A, Jaffe P, Efron J et al (2003) Colonoscopy: why are general surgeons being excluded? Surg Endosc 17:1971-1973

13. Sroka G, Feldman LS, Vassiliou MC et al (2010) Fundamentals of laparoscopic surgery simulator training to proficiency improves laparoscopic performance in the operating room-a randomized controlled trial. Am J Surg 199:115-120

14. Edelman DA, Mattos MA, Bouwman DL (2011) Impact of fundamentals of laparoscopic surgery training during medical school on performance by first year surgical residents. J Surg Res 170:6-9

15. Kolozsvari NO, Kaneva P, Vassiliou MC et al (2012) New dog, new tricks: trends in performance on the fundamentals of laparoscopic surgery simulator for incoming surgery residents. Surg Endosc 26:68-71

16. Okrainec A, Soper NJ, Swanstrom LL et al (2011) Trends and results of the first 5 years of fundamentals of laparoscopic surgery (FLS) certification testing. Surg Endosc 25:1192-1198

17. Derevainko AY, Schwaitzberg SD, Tsuda S et al (2010) Malpractice carrier underwrites fundamentals of laparoscopic surgery training and testing: a benchmark for patient safety. Surg Endosc 24:616-623 